\title{
Optimizing drum-boiler water level control performance: A practical approach
}

\author{
Ahmed El-Guindy, Simon Rünzi and Kai Michels \\ University of Bremen, Institute of Automation (IAT), 28359 Bremen, Germany
}

\begin{abstract}
This paper presents the development of a LQR to optimize pressure and water level control performance of a drum-boiler unit that belongs to a $450 \mathrm{MW}$ CCHP plant in Germany. A nonlinear model is initially built within MATLAB/Simulink environment, later enlarged to include the process PID-controllers and control valves regulating mass flow rates. The complete model is validated against data measurements from the plant with very rich excitation. The concluded simulation results adopting the newly proposed control strategy show, that the suggested multivariable control technique outperforms the existing PID-controller in many aspects improving the control performance significantly and yielding much tighter reference value tracking during load changes.
\end{abstract}

Index Terms-drum-boiler level control, LQR, multivariable feedback control, power plant simulation

\section{INTRODUCTION}

Energy market deregulation and the integration of renewable energy sources into the electrical grid have led to dramatic changes in the power industry, which escalated rapidly new challenges, that have to be met by conventional power plants. Such evolution caused a noticeable process modification regarding how load-following power plants operate, as they should become more flexible to fulfill their load requirements, which became more frequent nowadays.

The process controllers have to be designed in a way, that simultaneously fulfill the load demand as soon as possible, while at the same time bearing in mind safety and life span of the plant crucial elements. One common challenge is the steam drum-boiler unit control, supplying the steam turbine continuously with high pressure and temperature steam. The controller should maintain drum pressure and water level within acceptable limits over the complete operating range. If the level exceeds the upper limits, water would be carried over to the superheater or the turbine, leading to outage of either the turbine or the boiler. Surpassing lower limits would cause overheating of the water wall tube resulting in serious tube rupture and severe damage.

The industry standard 2-element or 3-element PIDcontroller used to regulate the process can be found in most of the power plants. They can behave fairly well with low load changes, however the performance becomes unsatisfactory for higher steps, resulting in large overshoots in the system response. Modifying the controller parameters to improve the system performance is quite a challenging task, since the steam generation process is a multivariable nonminimum phase system, having an initial inverse response which varies according to the operating point [1]. This behaviour is associated with the physical phenomena known as shrink/swell of steam bubbles under the water level.

Various modern control strategies for drum water level were proposed in literature in the recent years. We can mention robust control using $\boldsymbol{H}_{\infty}$ mixed-sensitivity [2] and $\boldsymbol{H}_{\infty}$ loop-shaping [3] approaches, predictive control method [4], adaptive techniques utilizing grey predictor based algorithm (GPBA) [5] and genetic algorithm [6], fuzzy logic [7] and nonlinear sliding mode control [8]. Even though the above mentioned approaches can manage to improve the control performance, as observed within different simulation environments, it can lack many practical aspects such as online tuning and ease of implement within a real plant distributed control system (DCS).

In this paper, we propose a multivariable feedback technique using an observer-based linear-quadratic regulator with integral action, keeping in mind tradeoff between controller complexity and sacrifice of its optimal robust performance, as the resulting controller scheme is structurally constrained. Switching between the existing controller and the LQR should be carried on easily, where the changes performed on the DCS existing hierarchical structure are kept minimal.

The work presented here is part of an ongoing research project taking place in collaboration with Munich City Utilities $\mathrm{GmbH}$ (SWM), in regards with the process controller of the low pressure drum-boiler unit, located within the $450 \mathrm{MW}$ combined cycle heat and power plant GuD 2 located at the combined heat and power facility HKW Süd.

\section{Process Modelling}

HKW Süd classified as a combined cycle cogeneration plant, can handle concurrent production of electrical power and useful heat, by utilizing a class of sustainable integrated technologies progressively being used. Exhaust gas emitted from the gas turbine can be reused as heat source for steam production, required to operate the steam turbine. Therefore more useful energy can be extracted, supplying additional electricity to the grid.

The simplified process shown in Fig. 1 illustrates steam production using the drum-boiler unit. Supplied water fed by the pump to the drum inlet is preheated through the economizer, in order to reduce energy consumption. Due to the gravity, water flows down through a downcomer-riser closed loop producing saturated steam, which flows along the riser tubes before being collected and fed back into the 
drum. The saturated steam flows through the water level until it exits upon reaching the drum outlet. Then it is reheated one more time at the superheater.

The inflow from the feedwater pump is regulated using one control valve. The steam leaving the drum can be regulated using five valves connected in parallel. In steady-state only one valve is operational while the others are closed, where each has its own distinctive construction and functionality.

1) The water tank control valve supplies a constant portion of steam for heating the feedwater tank.

2) The security valve is employed for safety matters to relief the pressure inside the drum.

3) The bypass valve supplies heat to city districts.

4) The condenser control valve bypasses the steam turbine, feeding directly the condenser.

5) The steam turbine control valve feeds the steam turbine, generating additional electricity.

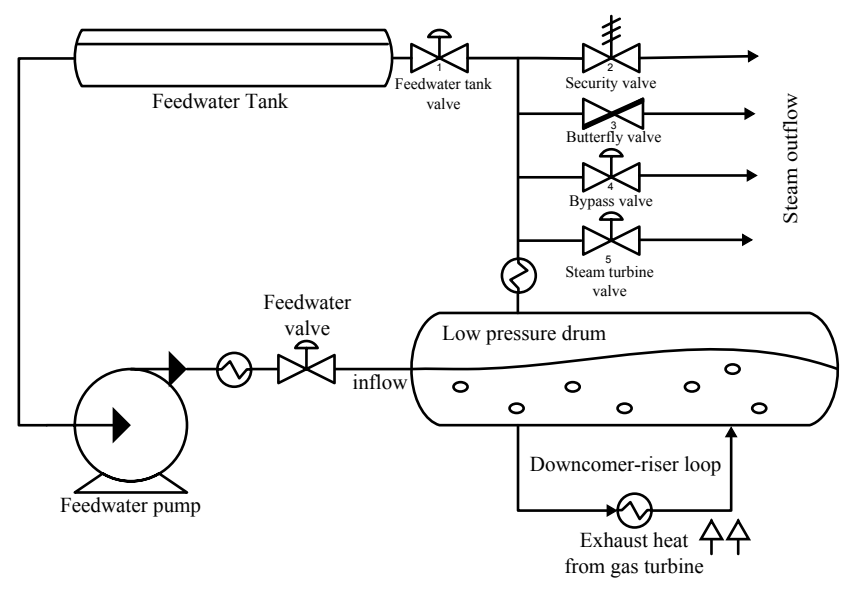

Fig. 1. Drum-boiler process description

\section{A. Drum-boiler model}

The drum complex geometry with riser and downcomer tubes and specially the two phase flow modelling is usually quite complicated, which requires typically usage of partial differential equations. The well developed Åström - Bell model [1] is being considered.

The majority of the system can be captured through a $4^{\text {th }}$ order nonlinear model, by means of defining mass flow and energy balance with the help of a physical mechanism introduced under the following elementary assumption.

Most of the system parts will be under thermal equilibrium due to their direct contact with saturated liquid/vapour mixture. The energy stored in the mixture is either absorbed or released quickly following drum pressure changes, meaning that various metal parts of the system would adapt their temperatures in the same manner. This agrees with experimental observation, which has proven, that the difference between both temperatures is very small, thus a detailed representation of the temperature distribution within the metal is not necessary.

The distribution of steam along the riser tubes was carried out using a lumped model, which represents the energy and mass balance caused by the naturally circulated downcomerriser closed loop. The steam mass ratio, assumed to vary linearly from the inlet to the outlet of the riser, is characterized in response to changes in the downcomer, riser and heat flow rates respectively.

Many of the complex phenomena in the drum can be captured by an empirical equation, which resulted from various attempts, over the years, to fit with the experimental data. It can capture most of the process dynamics by proper parameterizations of the model variables, namely, residence time of steam inside the drum $T_{d}$, the bubbles steam volume at hypothetical situation $V_{s d}^{\circ}$ and friction coefficient in downcomer-riser loop $k$.

\section{B. Drum-boiler nonlinear state equations}

The chosen state variables should have a good physical interpretation. Drum pressure $P$ is obviously chosen as it describes the total energy of the system. The water volume $V_{w t}$ in the system is selected since it represents the storage of mass. Steam quality $\alpha_{r}$ in the riser tubes and steam bubbles volume under the liquid level $V_{s d}$ are chosen as well to describe distribution of steam under the water, thus estimating the level.

To easily represent the equations, let $V$ represent volume, $\dot{Q}$ and $q$ denote heat and mass flow rates respectively, $h$ specific enthalpy, $P$ pressure. Additionally, the subscript $s$, $w, f, c$ refers to steam, water, feedwater and condensation.

The nonlinear algebraic state Eqs. (1), (2), (3) and (4) introduced in [1], resulted from straight forward calculations of the mass and energy balance for the drum, naturally circulated downcomer-riser loop and steam bubbles under water level respectively.

$$
\begin{aligned}
\dot{P} & =\frac{e_{11} \dot{Q}+q_{f}\left(e_{11} h_{f}-e_{21}\right)-q_{s}\left(e_{21}-e_{11} h_{s}\right)}{e_{11} e_{22}-e_{12} e_{21}} \\
\dot{V}_{w t} & =\frac{\dot{Q}+q_{f} h_{f}-q_{s} h_{s}-e_{22} \dot{P}}{e_{21}} \\
\dot{\alpha}_{r} & =\frac{\dot{Q}-\alpha_{r} h_{c} q_{d c}-e_{31} \dot{P}}{e_{33}} \\
\dot{V}_{s d} & =\frac{1}{T_{d}}\left(V_{s d}^{\circ}-V_{s d}\right)-q_{f} \frac{h_{f}-h_{w}}{e_{44} h_{c}}-\frac{e_{41}}{e_{44}} \dot{P}-\frac{e_{43}}{e_{44}} \dot{\alpha}_{r}
\end{aligned}
$$

The resulting nonlinear model states are $\boldsymbol{x}=\left[P, V_{w t}\right.$, $\left.\alpha_{r}, V_{s d}\right]$. The actuating variables $\boldsymbol{u}=\left[q_{f}, q_{s}\right]$ are the feedwater and steam flow rates, manipulated to control the model outputs $\boldsymbol{y}=[P, l]$.

The heat flow rate $\dot{Q}$ is rather considered as a model input disturbance $z$ due to the fact that its amount is associated with the gas turbine exhaust heat, which in return corresponds to its electrical output power. On the contrary heat flow rate becomes a control variable in thermal plants as it can be regulated directly by adjusting the boiler firing rate.

\section{Control valves model}

The modelling of feedwater and steam mass flow rates regulation through a control valve, which meets practical needs, can be achieved using Eqs. (5) and (6). They are 


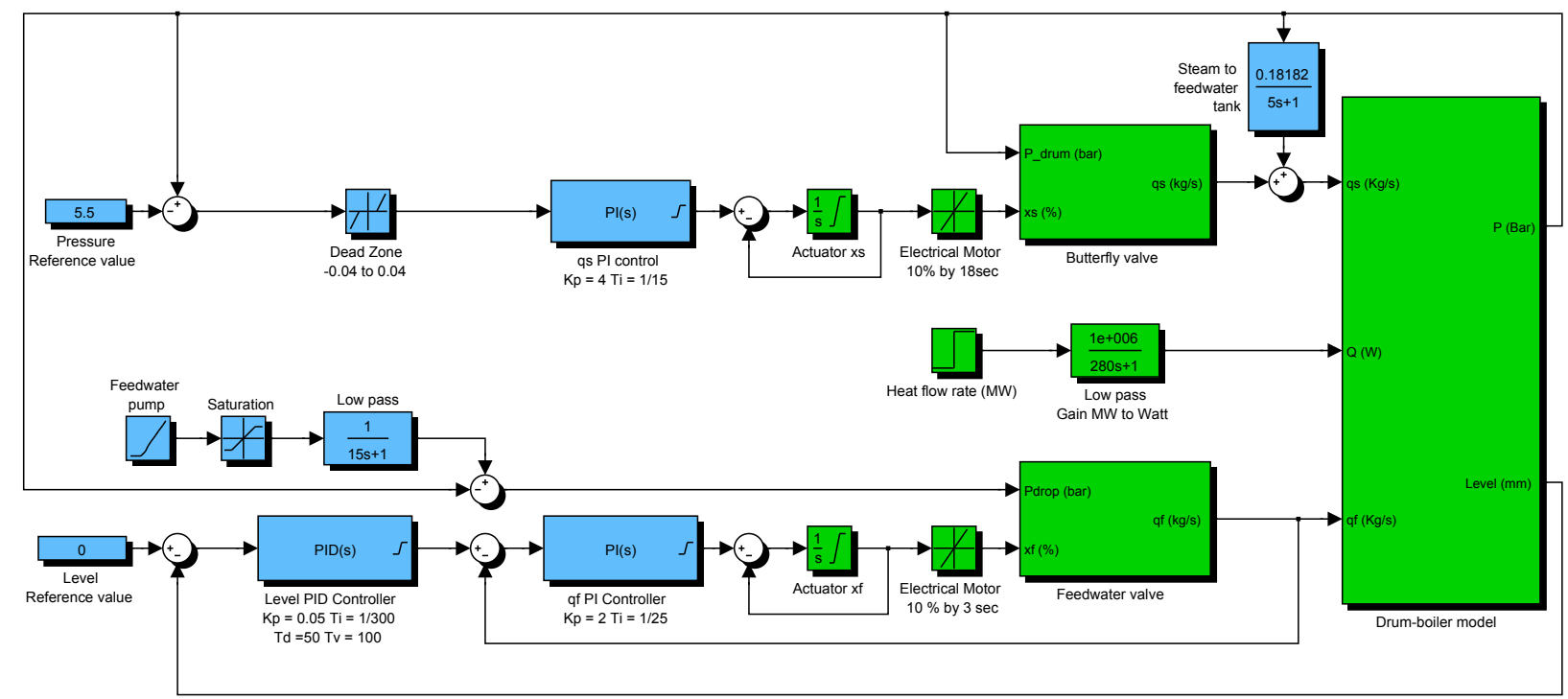

Fig. 2. Process with existing family of PID controllers

essentially used by mechanical engineers to size their valves and meet mass flow requirements.

The pressure drop $\Delta P$ across the valve is the difference between the feedwater pump and drum pressures, $K_{v}$ is the valve sizing coefficient, and finally, $x$ is the valve percentage opening ranging from $0 \%$ to $100 \%$, where the actuator used to operate it, is modeled as a $1^{\text {st }}$ order lag element in series with a rate limiter.

$$
\begin{aligned}
& q_{f}=x_{f} \cdot \frac{K_{v f} \rho_{w} \sqrt{\Delta P}}{3600} \\
& q_{s}=x_{s} \cdot \frac{13.6 \sqrt{\rho_{s} P} K_{v s}}{3600}
\end{aligned}
$$

\section{Water level and pressure controllers}

The drum pressure and water level set values are always kept constant regardless of the heat flow rate supplied amount. Each output is controlled with its separate decoupled control loop, without considering any sort of interaction between both outputs.

The level controller currently implements the 2-element structure, which employees a cascaded control architecture using level and feedwater flow rate as process variables. The outer loop compares the current level with the specified reference, the computed error signal generates using a PIDcontroller the new set value for feedwater flow rate. The inner loop examines the current flow with the amount established by the outer loop, in order to adjust accordingly the control valve percentage opening using a PI-controller.

During normal operation, the pressure is regulated by modifying the steam turbine or bypass valves position using identical control loop structures. It consists of a simple feedback loop, which compares the reference value with the actual pressure before being altered using a PI-controller.

\section{VALIDATION}

The system closed loop response will be validated and examined against data from the real plant for different scenarios, to experiment its ability to capture the real process dynamics at various operating conditions. The complete model with the PID-controllers is shown in Fig. 2.

\section{A. Assumptions}

The heat flow rate required as an input of the model is predicted from the gas turbine electrical output power. The transfer function relating both is assumed to be $1^{\text {st }}$ order lag element, whose time constant was identified based on the assumption, that the supplied heat behaviour is directly associated with the evaporator temperature.

The feedwater valve position is always kept half-way opened in the plant without considering the amount of feedwater which flows through it. Therefore the pressure drop across the valve should increase or decrease accordingly to preserve such condition, which is achieved using the feedwater pump controller. Implementation of the controller was neglected for simplicity.

The tests conducted in the plant were using only the butterfly valve to regulate the steam flow rate. Therefore the pressure control loop will consist only of the corresponding PI-controller.

\section{B. Comparison with measurement data}

The pressure controller senses the pressure decrease within the drum as less heat is being supplied as shown in Fig. 3, thus it tries to close the butterfly valve to restore pressure back to its set point. Once the valve starts closing, the water level $l$ drops due to the shrinking effect of steam bubbles. It experiences an undershoot followed by an overshoot, since the cascade controller is simultaneously trying to restore the level back to its set point and to reestablish energy balance for the drum, as well as mass balance for inflow and outflow. 
Fig. 4 illustrates the comparison considering gas turbine power increase. The controllers react on the pressure rise within the drum caused by the additional heat supplied, therefore opening the corresponding valve to relief drum pressure allowing more steam to leave from the drum outlet in the process. The water level $l$ increases due to steam bubbles swelling, yet a smaller overshoot is observed since the change of electrical power is less when compared to the previous scenario, thus in return permit the controllers to settle and drive the process back to steady-state faster.

The comparison results show that the model can capture the drum dynamics to a great extent. However, a relatively small deviation from real measurements is still noticeable. The error arises due to the assumptions made in order to simplify the complete process simulation. Nevertheless, the model current attitude can be regarded as satisfactory, bearing in mind, that several control loops were omitted for simplification. Eventually, a realization within the real plant would ultimately require an observer gain, to correct the states based upon the difference between real measurements and model outputs.
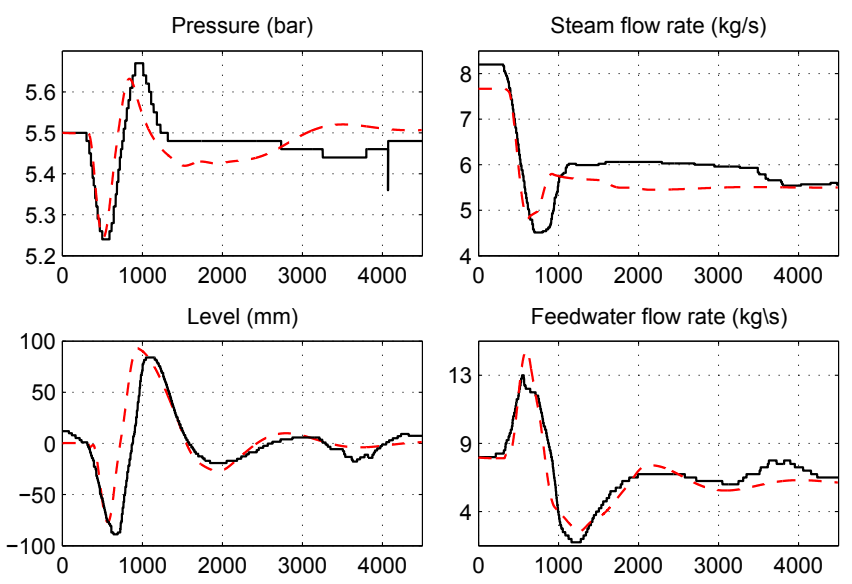

Fig. 3. Comparison between model (Dashed) and plant (Solid) closed loop responses for a decrease of the gas turbine power equivalent to $20 \mathrm{MW}$

The identified main problem with the existing level control can be outlined as follows. Assuming drop of exhaust heat provided to the drum leads to decrease of pressure and water level. The feedwater control valve supplies more water through the inlet, yet unintentionally contributing into additional drop of the level. From the physical point of view this takes place since the cold water fed into the drum decreases its temperature and as result its pressure as well. The pressure controller tries to close the steam valve even more to track the set value, leading eventually to further water drop due to steam bubbles shrinking.

\section{Optimal CONTROLLER DESigN}

The results observed during system modelling, analysis and validation suggest, that an optimization of the process is achievable using a multivariable control technique. The strategy would account for synergy between feedwater and steam flow rates instead of just decoupling the MIMO system
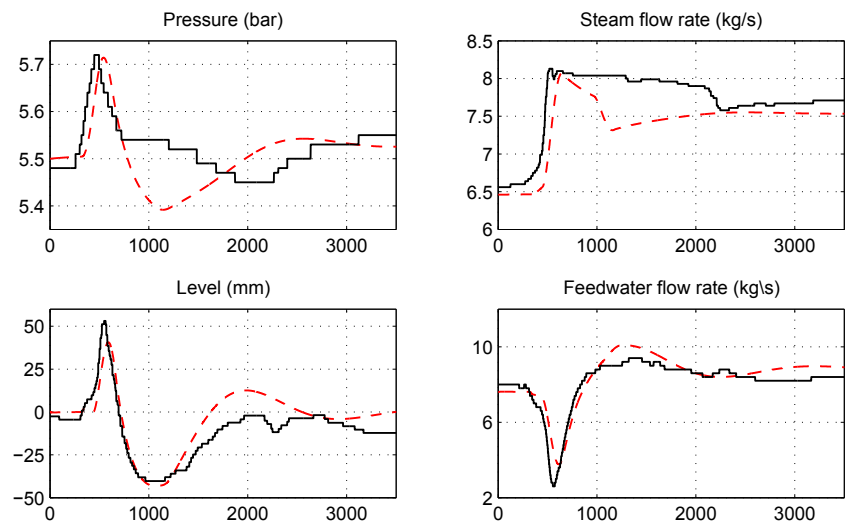

Fig. 4. Comparison between model (Dashed) and plant (Solid) closed loop responses for an increase of the gas turbine power equivalent to $10 \mathrm{MW}$

into several decoupled SISO systems regulated by their own noninteracting control loops.

A state-feedback controller, realized using a linearquadratic regulator (LQR), is suggested in order to consider the internal variables of the system instead of the process outputs, therefore accounting for additional aspects, discarded using the classical control methodology. The inner dynamics of the drum-boiler unit correspond to the developed nonlinear model state variables, defined in Sec. II-B.

In order to compute the controller and observer gain matrices, a linear model of the plant is required. The developed nonlinear model was linearized using MATALB Control System Toolbox ${ }^{\mathrm{TM}}$ linear time-invariant (LTI) functions, at the common operating point of the gas turbine, which is $85 \mathrm{MW}$ electrical output power.

\section{A. Linear-Quadratic regulator}

The LQ method is a significant result of the optimal control theory, which manages problems associated with quadratic performance criteria for state-space systems. It allows trade off between regulation performance and control effort compared with the pole placement method. The resulting optimal feedback gain $\boldsymbol{K}$ should drive the closedloop system without external input from any initial state to the zero state minimizing the cost function Eq. (7).

$$
J=\int_{0}^{\infty}\left(\boldsymbol{x}^{T}(t) \boldsymbol{Q} \boldsymbol{x}(t)+\boldsymbol{u}^{T}(t) \boldsymbol{R} \boldsymbol{u}(t)\right) \mathrm{d} t
$$

$\boldsymbol{Q}$ and $\boldsymbol{R}$ matrices are positive definite matrices assigned as weighting factors for the course of states and input variables. Faster convergence of a particular state towards zero should increase its equivalent coefficient inside the matrix $Q$. If a slower response of the actuating variables is preferred to lower the energy consumption and minimize control effort, then coefficients of $\boldsymbol{R}$ matrix have to be chosen larger. Choosing the values of $\boldsymbol{Q}$ and $\boldsymbol{R}$ matrices, in principle, is similar to tuning of PID-controller parameters, the weighting matrices are varied until a satisfactory response is reached.

A reasonable simple choice for the matrices $\boldsymbol{Q}$ and $\boldsymbol{R}$ is given by the Brysons rule, it scales the variables appearing in 


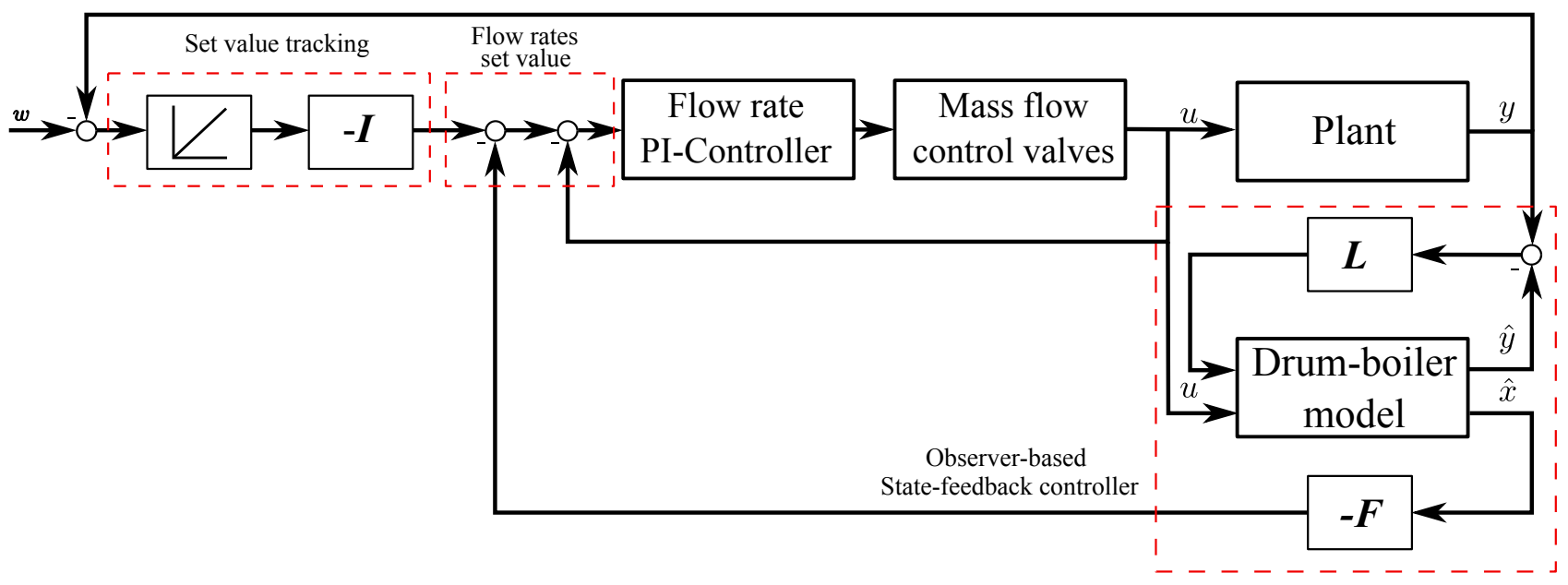

Fig. 5. Proposed control strategy

Eq. (7) so that the maximum acceptable value of each term is one [9]. Setting the drum-boiler model states limits was straight forward and helped performing the initial simulation. The drum pressure limit would be 9 bar as specified by construction data, the error between reference and actual measurement shouldn't exceed 0.3 bar. The water level maximum allowable deviation from set point isn't allowed to surpass $\pm 150 \mathrm{~mm}$.

The main challenge lies in stabilization the system, while considering the limitations and constraints imposed by the control valves position range and opening/closing fixed rate of change. Several simulations took place varying mainly coefficients associated with mass flow rates, pressure and level error signals respectively, until good results were obtained.

\section{B. Luenberger observer}

The drum-boiler unit nonlinear model is already estimating drum-boiler unit, however without feedback of the observer gain matrix $\boldsymbol{L}$. It shall be designed to eliminate the error between measured data and model outputs, noticed during validation in Sec. III. The observer gain isn't calculated using Kalman filter because efficient practice of the method requires continuous testing of the estimator alongside the real process. Further, the optimal choice of the filter weighting matrices depend on reliable prediction of the process noise, which is only guaranteed by regular observation.

The pole placement method is applied despite its disadvantages with respect to the following practical consideration. The observer response must be faster than the closed loop employing the state-feedback controller, as the estimation error has to decay to zero causing the state variables to converge, before they can be used for control. As a rule of thumb, the slowest observer pole should be five times faster than the fastest pole of the LQR.

\section{Simulation RESUlts}

The simulated optimized system performance will be shown in the following figures. First we shall examine the estimated states when employing the observer gain along the nonlinear model, to check if the new pole positions introduce noise into the system. Then the model behaviour with state correction is validated against new measurement data with very rich excitation, covering the drum-boiler operating range. Therefore the observer stability can be investigated, providing a good indication of the proposed control strategy applicability, since the states are crucial for feedback.

The comparison between both controllers is addressed to check if the newly proposed state-feedback controller handles efficiently the main problems identified with the current controller. Further, the states and flow rates at different load conditions are inspected to ensure stability of the linear statefeedback matrix $\boldsymbol{K}$ for the entire drum operating range.

\section{A. Observer performance subjected to perturbations in gas turbine electrical output power}

Fig. 6 and Fig. 7, show the comparison between the observer outputs and states against the real process when the electrical power of the gas turbine was switched in between $110 \mathrm{MW}$ and $70 \mathrm{MW}$ for approximately two hours. In addition, Fig. 6 shows the observer behaviour using various state estimator gains computed at different pole positions. Significant improvement of the model closed loop response when combined with the observer gains was achieved.

\section{B. State controller performance subjected perturbations in gas turbine electrical output power}

The drum pressure and water level were vastly enhanced when analyzing both behaviours, depicted in Fig. 8. The level maximum peak overshoot/undershoot didn't exceed $\pm 100 \mathrm{~mm}$ during transients, and the pressure never surpasses the safety limits, which might lead to operation of the security valve.

Obviously, the steam flow rate performance is quite similar using both controllers, but the feedwater flow rate behaviour was modified significantly. This is no surprise and should 
have been expected following process analysis which diagnosed the drum level cascade controller and highlighted its particular weakness. The optimal state controller managed the initial inverse response, caused by the shrink/swell physical phenomena, when considering the inner dynamics of the system instead of the outputs.

\section{CONCLUSIONS}

A mathematical nonlinear model, which describes the dynamical process of steam generation using steam drumboiler units including its control valves and process PIDcontrollers, was fitted to a real drum-boiler unit which corresponds to $450 \mathrm{MW} \mathrm{CHP}$ in Munich, in order to analyse the pressure and water level control performance, reported to behave very poorly during transients corresponding to large load changes.

The model was implemented within MATLAB/Simulink environment and examined intensively throughout various scenarios with very rich excitation from the plant covering a wide operating range, to ensure its validity and reliability. Further it pointed out very clearly the main drawbacks of the existing control strategy.

A multivariable feedback control strategy is proposed in order to optimize the process using an I-based statefeedback controller designed using LQ method, ensuring steady-state accuracy and set value tracking. Additionally an observer gain, which guarantees correct estimation of the state variables required for feedback, is realized using pole placement method. Simulation results show that the statefeedback controller outperforms the PID-control in terms of control behaviour and performance.

From a practical perspective, the proposed strategy can be integrated in parallel with the existing controller, without many modifications within the DCS. The plant personal would easily switch between mass flow rates set values, generated by either the decoupled PID-controller or the LQR.

At the moment, the nonlinear model was successfully implemented in the "Mauell - Process Control System ME4012" at HKW Süd to act as an observer of the process, thus offering in return a great opportunity to test and examine the model more closely, before being combined with the LQR.

\section{REFERENCES}

[1] Åström, Karl Johan and Bell, Rodney D., "Drum Boiler Dynamics", Automatica, 36, 363-378, 2000.

[2] G. Pellegrinetti and J. Bentsman, " $\boldsymbol{H}_{\infty}$ controller design for boilers", International Journal of Robust and Nonlinear Control, 4:645-671, 1994

[3] Tan, W., Marquez, H. J., Chen, T. and Gooden, R. K., " $\boldsymbol{H}_{\infty}$ control design for an industrial boiler", Procceeding of ACC Conference, 2537-2542, 2001.

[4] M. Xu , S. Li and W. Cai, "Cascade generalized predictive control strategy for boiler drum level", ISA Trans., 44(3):399-411, 2005.

[5] N. Yu, W. Ma, M. Su, "Application of adaptive grey predictor based algorithm to boiler drum level control", Energy Conversion and Management, 47:2999-3007, 2006.

[6] Y. Nanhua, M. Wentong and S. Ming, "Boiler-Turbine Control System Design Using a Genetic Algorithm", IEEE Transactions on Energy Conversion, 10(4):752-759, 1995
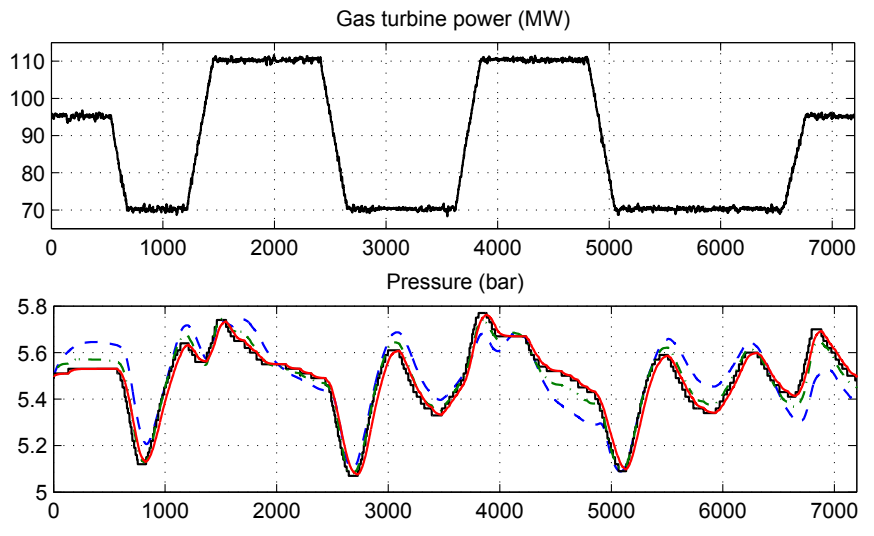

Fig. 6. Observer behaviour comparison for perturbations in gas turbine electrical output power
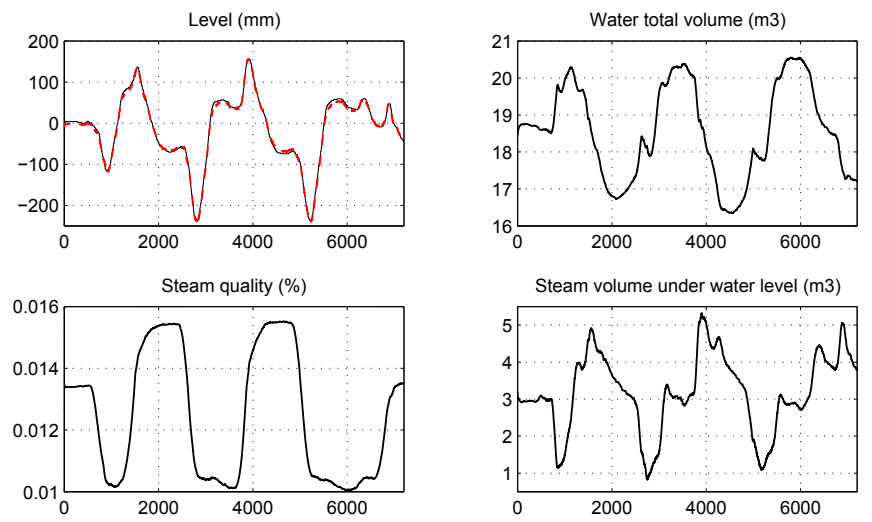

Fig. 7. Comparison between state observer (dashed) and plant (solid line) for perturbations in gas turbine electrical output power
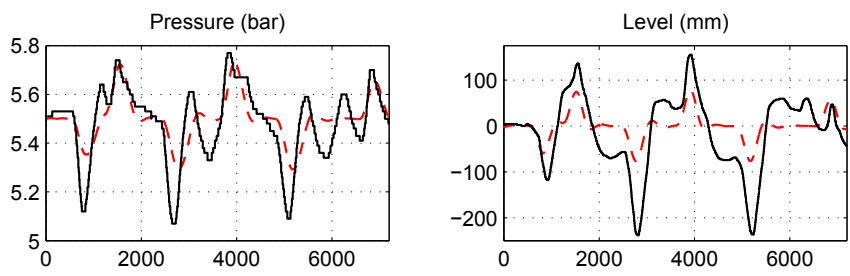

Steam flow rate $(\mathrm{kg} / \mathrm{s})$
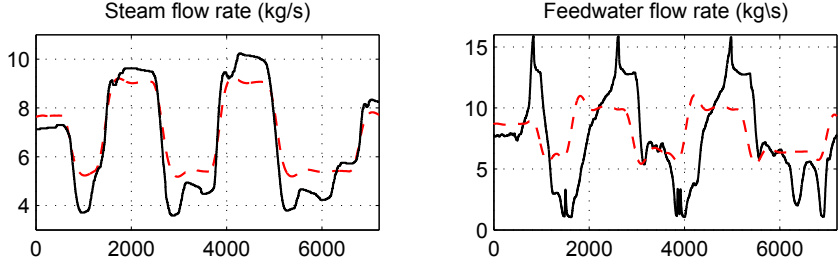

Fig. 8. Comparison between the LQR (dashed) and old controller (solid line) for perturbations in gas turbine electrical power

[7] Vue Wei-jie, Liu Yong-xin, "Boiler Drum Level controlled by Fuzzy Self-adapting PID”, Second Asia-Pacific Conference on Computational Intelligence and Industrial Applications, 2:381-384, 2009.

[8] H. Moradi, M. Saffar-Avval, F. Bakhtiari-Nejad, "Sliding mode control of drum water level in an industrial boiler unit with time varying parameters: a comparison with H-infinity robust control approach", Journal of Process Control, 22:1844-1855, 2012.

[9] J. P. Hespanha, "Lecture notes on LQR/LQG controller design", University of California, Santa Barbara, 2005. 